\title{
Quantitative Imaging in Pediatric Hepatobiliary Disease
}

\author{
Haesung Yoon, MD, PhD, Hyun Joo Shin, MD, PhD, Myung-Joon Kim, MD, PhD, Mi-Jung Lee, MD, PhD \\ All authors: Department of Radiology, Severance Hospital, Severance Pediatric Liver Disease Research Group, Research Institute of Radiological \\ Science, Yonsei University College of Medicine, Seoul, Korea
}

Pediatric hepatobiliary imaging is important for evaluation of not only congenital or structural disease but also metabolic or diffuse parenchymal disease and tumors. A variety of ultrasonography and magnetic resonance imaging (MRI) techniques can be used for these assessments. In ultrasonography, conventional ultrasound imaging as well as vascular imaging, elastography, and contrast-enhanced ultrasonography can be used, while in MRI, fat quantification, T2/T2* mapping, diffusion-weighted imaging, magnetic resonance elastography, and dynamic contrast-enhanced MRI can be performed. These techniques may be helpful for evaluation of biliary atresia, hepatic fibrosis, nonalcoholic fatty liver disease, sinusoidal obstruction syndrome, and hepatic masses in children. In this review, we discuss each tool in the context of management of hepatobiliary disease in children, and cover various imaging techniques in the context of the relevant physics and their clinical applications for patient care.

Keywords: Child; Liver; Ultrasonography; Elasticity imaging techniques; Magnetic resonance imaging

\section{INTRODUCTION}

Pediatric hepatobiliary imaging is important for evaluation of congenital or structural disease as well as metabolic or diffuse parenchymal disease and tumors. A range of imaging modalities, including ultrasonography, magnetic resonance imaging (MRI), and computed tomography (CT) can be used for these purposes. In ultrasonography, echotexture analysis can be performed on conventional grayscale images. Both conventional Doppler imaging and advanced microvascular imaging can evaluate tissue vascularity and perfusion. Ultrasound (US) elastography can evaluate tissue stiffness, while contrast-enhanced ultrasonography (CEUS) can determine hemodynamic changes in pathologic conditions. Various sequences can be used to quantify hepatobiliary

Received January 2, 2019; accepted after revision June 11, 2019. Corresponding author: Mi-Jung Lee, MD, PhD, Department of Radiology, Severance Hospital, Severance Pediatric Liver Disease Research Group, Research Institute of Radiological Science, Yonsei University College of Medicine, 50-1 Yonsei-ro, Seodaemun-gu, Seoul 03722, Korea.

- Tel: (822) 2228-7400 - Fax: (822) 393-3035

-E-mail:mjl1213@yuhs.ac

This is an Open Access article distributed under the terms of the Creative Commons Attribution Non-Commercial License (https://creativecommons.org/licenses/by-nc/4.0) which permits unrestricted non-commercial use, distribution, and reproduction in any medium, provided the original work is properly cited. disease in MRI, including fat quantification, $\mathrm{T} 2 / \mathrm{T} 2$ * mapping, diffusion-weighted imaging (DWI), magnetic resonance (MR) elastography, and dynamic contrastenhanced (DCE) MRI. Separately, even though ionizing radiation exposure is a concern, advanced $\mathrm{CT}$ techniques such as dual-energy CT (DECT) can differentiate between materials by using multi-material decomposition algorithms.

With these techniques, quantitative imaging can enhance the diagnostic accuracy for hepatobiliary diseases. Moreover, quantitative imaging results can be used to determine disease status and evaluate treatment response and followup. In this review, we present the use of each tool in the management of hepatobiliary disease in children, including discussions on the imaging techniques along with the relevant physics and clinical applications for patient care.

\section{Imaging Techniques in Ultrasonography}

Ultrasonography is the first modality of choice for imaging assessment of hepatobiliary disease in infants and children due to its widespread availability, easy accessibility, high resolution, and lack of the need for sedation.

\section{Conventional Ultrasonography}

The normal echotexture of the hepatic parenchyma in the pediatric liver generally does not differ from that 
seen in normal adult livers. Normal liver echogenicity is homogeneously low to intermediate and similar to that of the renal cortex or spleen, and the peripheral portal venous vasculature can be clearly seen (1). In cases with hepatic fat infiltration, increased liver echogenicity, a lack of distinction between vessels and bile ducts, posterior beam attenuation, poor diaphragm visualization, and a tightly packed echo pattern are observable (2). However, it is not always easy to quantify these imaging findings. This method has low accuracy with high variability and limitations in patients with high body mass indices.

The common bile duct should measure less than $1 \mathrm{~mm}$ in neonates, less than $2 \mathrm{~mm}$ in infants up to one year of age, less than $4 \mathrm{~mm}$ in older children, and less than $7 \mathrm{~mm}$ in adolescents and adults (1). In cases of hepatobiliary disease, splenomegaly can appear as a secondary finding. In children, the standard value for a normal spleen size increases with age, height, and weight (3).

\section{Ultrasonographic Vascular Imaging}

Doppler imaging employs the Doppler effect to generate images of the movement of fluids such as blood, and the relative velocity to the probe can be calculated by determining the frequency shift of a particular sample volume. A recent US microvascular imaging technique, superb microvascular imaging (SMI), has been designed for detection of subtle low-flow components (4). Both blood flow and tissue motion, called "clutter," produce ultrasonic Doppler signals. Conventional Doppler ultrasonography uses a wall filter to remove clutter and motion artifacts, resulting in the loss of low-flow components. However, in comparison, SMI can separate flow signals from underlying clutter by using an adaptive algorithm.

\section{US Elastography}

US elastography employs ultrasonic echoes to observe tissue displacement as a function of time and space after application of a force. The displacement measurements can be performed using three approaches: 1) tissue displacement may be displayed directly, as in acoustic radiation force impulse (ARFI) imaging; 2) tissue strain may be calculated from the spatial gradient of displacement and displayed, producing what is known as strain elastography; or 3 ) when the force is dynamic only, the time-varying displacement data may be used to record the arrival times of propagating shear waves at various positions (5). Among these, different shear wave technologies have been developed, including transient elastography (FibroScan ${ }^{\circledR}$, Echosens, Paris, France), point shear wave elastography (SWE), and two-dimensional (2D) or three-dimensional (3D) SWE. SWE uses sound waves to interrogate the mechanical stiffness properties of tissues and provides quantitative information on shear wave propagation.

When applying this technique in children, the use of different probes and acquisition depths to account for variable body sizes $(6,7)$ and optimal acquisition numbers to reduce the scan time as much as possible should also be considered. Three acquisitions may be sufficient for hepatic shear wave velocity measurements in children older than six years regardless of breathing status; however, more acquisitions are recommended for children younger than five years (8). To enable a comparison between different US elastography techniques, the interconversion of elasticity measurements is also required (9). For an example, a phantom elasticity of $13.0 \mathrm{kPa}$ can be considered as 8.8 $\mathrm{kPa}$ on the 1-6-MHz convex transducer of 2D SWE and 5.9 $\mathrm{kPa}$ on the $\mathrm{M}$ probe of transient elastography by using the reported equations.

The reported normal liver elasticity value using 2D SWE in children including newborns to 16 -year-olds in one study was $4.29 \pm 0.59 \mathrm{kPa}$ (range: $2.7-5.76 \mathrm{kPa}$ ) (10). This prior investigation revealed that liver elasticity had a significant nonlinear association with age, since the values in neonates and infants as well as adolescents were significantly higher than those in children aged 2-12 years. A previous study using ARFI also demonstrated that the liver shear wave velocity changed with age in children older than 10 years (11). In clinical applications, however, liver shear wave velocity is often increased and elasticity is diminished because of variable conditions, including fibrosis, inflammation, congestion, portal hypertension, and cholestasis.

\section{Contrast-Enhanced Ultrasonography}

CEUS is a safe and highly sensitive imaging modality that does not involve radiation exposure to the patient or operator and can be used for evaluation of focal liver lesions, intra-abdominal parenchymal injuries, and organ transplantations in children $(12,13)$. Safety considerations regarding the intravenous use of US contrast agents in children have primarily focused on "off-label" usage, although a large cohort study of adults demonstrated a comparable adverse events rate $(<0.01 \%)$ to that seen with MRI procedures (13). The recommended dose of sulfur 
hexafluoride gas containing contrast in children is based on body weight, as follows: $0.03 \mathrm{~mL} / \mathrm{kg}$ as an intravenous injection, up to a maximum of $2.4 \mathrm{~mL}$ per injection.

CEUS has shown comparable performance to that of CT and MRI in the pediatric work-up of focal liver lesions and blunt abdominal trauma in a systematic review (14). However, only a few studies have been conducted to date regarding the assessment of pediatric diffuse liver disease using CEUS. An animal study of rabbits demonstrated the feasibility of CEUS in evaluating liver fibrosis from biliary obstruction since the peak signal intensity of the liver was significantly associated with hepatic fibrosis grade (odds radio $=1.392 ; 95 \%$ confidence interval $[$ CI $], 1.114-1.741 ; p$ $=0.004)$ (Fig. 1) (15). This may indicate that CEUS can be used in liver fibrosis patients. However, further evaluation is needed.

\section{Imaging Techniques in Hepatobiliary MRI}

\section{Fat Quantification}

Measurement of the MR proton density fat fraction (PDFF) is a widely used method to quantify the amount of fat in the liver by using chemical shift gradient-recalled echo (GRE) sequence (16). Chemical shift imaging employs differences in precession frequencies in water and fat protons (17). In opposed-phase (OP) images, a difference in frequencies between water and fat causes a signal reduction, while inphase (IP) images demonstrate increased signal intensities as a result of the addition of water and fat signals. By comparing these IP and OP images, the fat fraction can be quantified by using the following equation: fat fraction $(\%)=([I P-0 P] /[2 \times$ IP] $] \times 100$ (18). Application of a fast GRE technique in chemical shift imaging, as in a modified Dixon method, offers benefits due to its rapid image acquisition, capability for use in breath-holding, and decreased misregistration (17). The cutoff values for diagnosing hepatic steatosis using PDFF are $5 \%$ in adults and $6 \%$ in children $(17,18)$.

\section{T2 or T2* Mapping}

T2 or T2* imaging is the most-used hepatic iron quantification method that employs MRI. Iron accelerates $\mathrm{T} 2$ relaxation and $\mathrm{T} 2$ * signal decay due to its paramagnetic properties in the liver. The heterogeneously distributed iron creates focal magnetic field inhomogeneity, which causes precession of protons at different frequencies and results in dephasing and signal decay. The greater the tissue iron levels, the smaller T2 and T2* become (19). R2 or R2* values are preferred because they directly correlate with iron concentrations and increase linearly with iron concentration, as determined by liver biopsy (20). R2* is more often used versus $\mathrm{R} 2$, since it can be measured in a single breath-hold and is less sensitive to variations in the size and distribution of iron particles (Fig. 2) (21).

Hepatic iron overload is the abnormal and excessive intracellular accumulation of iron in liver cells and is caused by excess intestinal absorption or repeated blood transfusions. This is problematic because free intracellular iron can cause cellular damage and may lead to fibrosis and cirrhosis in the liver (22). Conditions associated with

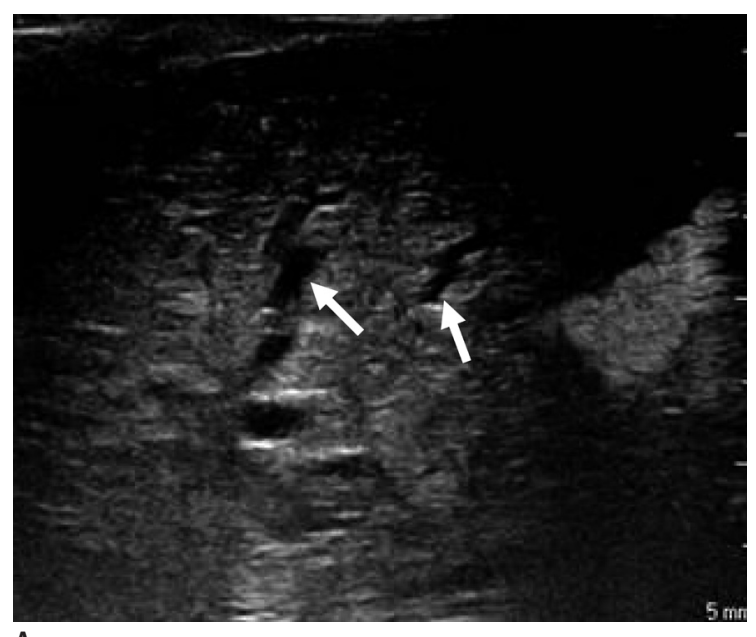

A

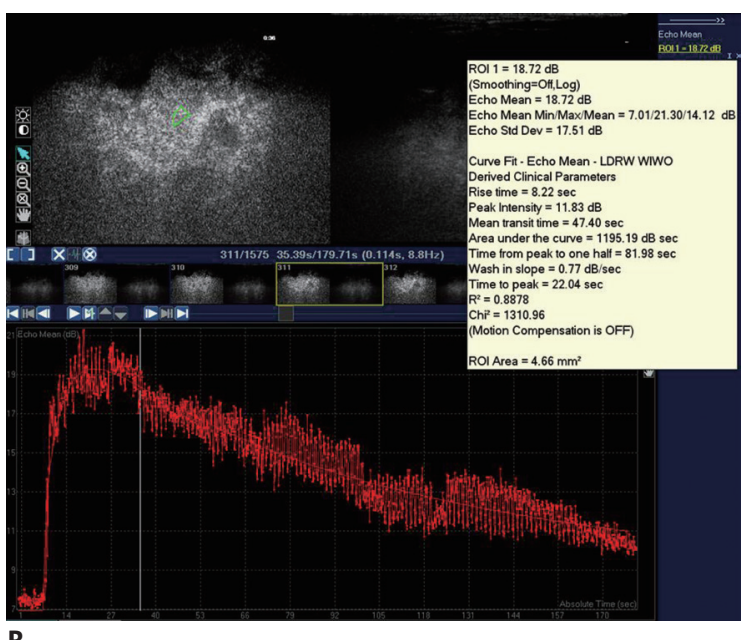

B

Fig. 1. Animal model of biliary obstruction for hepatic fibrosis evaluation.

A. Liver ultrasonography after bile duct ligation in rabbit shows diffuse intrahepatic bile duct dilatation (arrows). B. Contrast-enhanced ultrasonography of liver shows perfusion curve of liver parenchymal enhancement. Peak signal intensity was $11.83 \mathrm{~dB}$ in this case and hepatic fibrosis grade was 2. LDRW WIWO = local density random walk wash in wash out, ROI = region of interest 


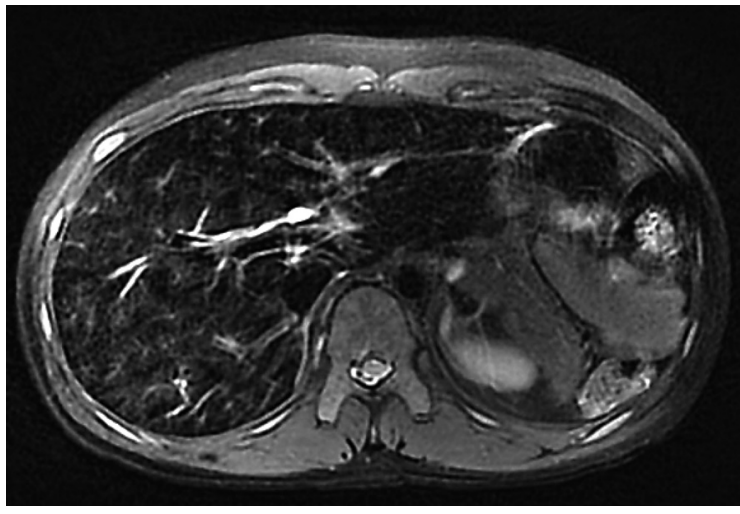

A

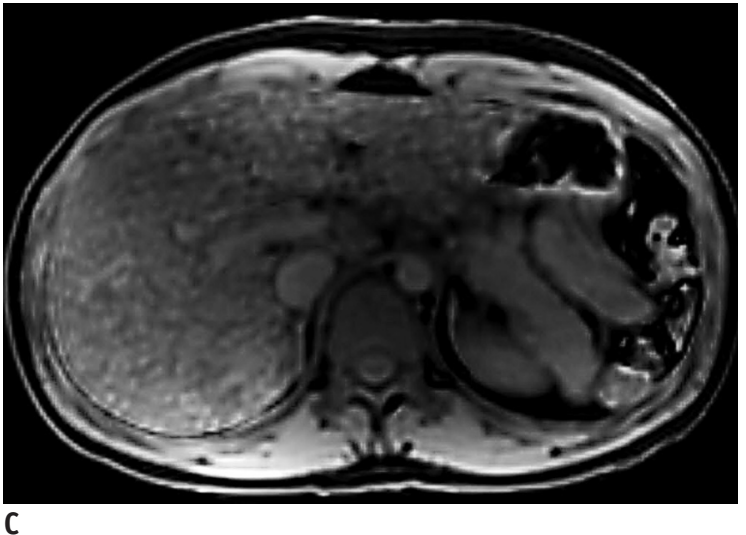

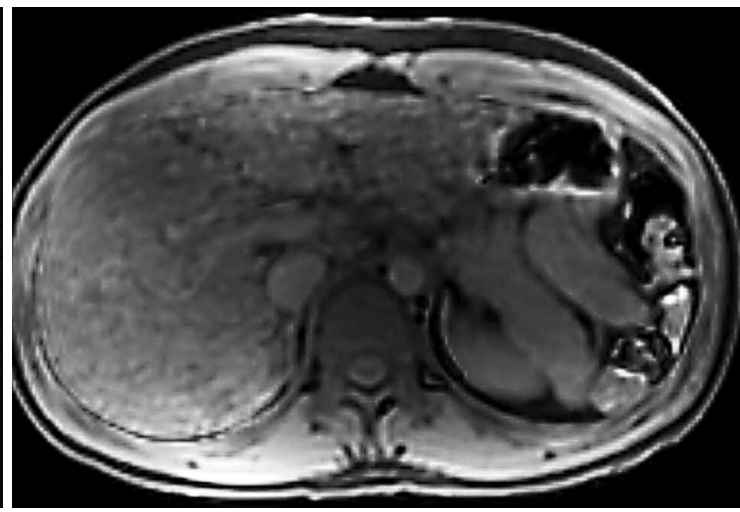

B

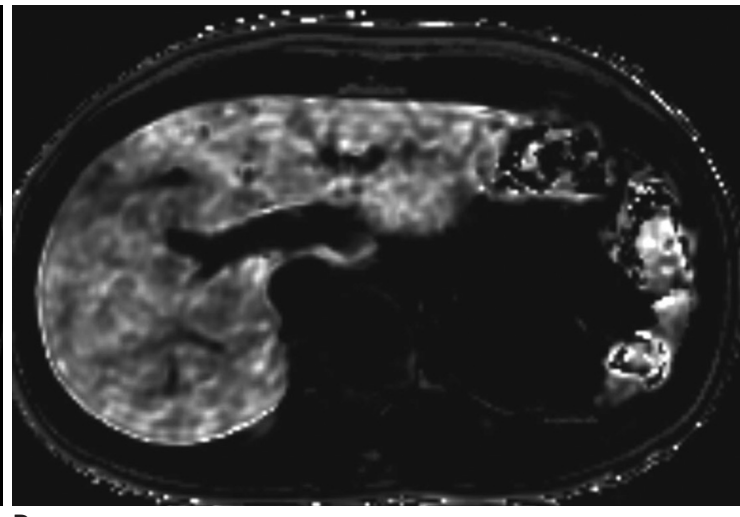

D

Fig. 2. 16-year-old male with hemolytic anemia and diffuse siderosis after splenectomy.

A. Axial T2-weighted MR image shows diffusely decreased T2 signal intensity of liver parenchyma. In-phase (B) and opposed-phase (C) T1weighted gradient-recalled echo images show no remarkable signal decrease on opposed-phase image. D. R2* parametric map demonstrates mild degree of iron overload in liver with R2* value of $329 \mathrm{~s}^{-1}$ at 3T MRI. MR = magnetic resonance

hepatic iron overload include hereditary hemochromatosis, thalassemia, sickle cell disease, chronic hepatopathy, and transfusional and parenteral iron overload. T2* imaging can accurately measure parameters adequate for both the diagnosis and treatment as well as follow-up of these diseases, even at low iron concentrations (23).

In the case of 1.5T MRI, a T2* value below $16 \mathrm{~ms}$ or R2* value over $60 \mathrm{~s}^{-1}$ is accepted as indicating hepatic iron overload, while a T2* value of $4 \mathrm{~ms}$ or R2* value of 270 $\mathrm{s}^{-1}$ discriminates moderate to severe hepatic iron overload from absent to mild hepatic iron overload (24). Relaxation rates (R2 and R2*) increase with field strength and liver R2* values measured with $3 \mathrm{~T}$ were almost double those observed at $1.5 \mathrm{~T}$ (25). High field strengths may be useful in detecting low degrees of iron overload but may present difficulties in the quantification of heavy iron overload (19).

\section{Mono-, Bi-, and Stretched Exponential Models DWI}

DWI quantifies water molecular motion using gradient pulse sequences (26). Usually, a single-shot spin-echo
T2-weighted image is used and two symmetric diffusion gradients are applied before and after a $180^{\circ}$ refocusing pulse (27). In restricted diffusion, phase shifts of spins after two diffusion gradients occur in the same direction, causing increased signal intensity. In free diffusion, phase shifts of spins will be held in different directions and this could lead to decreased signal intensity after application of two diffusion gradients (28). Conventionally, DWI and apparent diffusion coefficient (ADC) are measured from a mono-exponential model (27).

A bi-exponential model or intravoxel incoherent motion (IVIM) contends that the mono-exponential model is influenced by perfusion-related diffusion and true water molecular diffusion (29). Fast diffusion ( $D^{*}$, pseudodiffusion), representing relative blood flow, and the perfusion fraction (f), which represents relative blood volume, can be measured from the slope in low b-values. Slow diffusion ( $D$, true diffusion) can be calculated from the slope in high $b$-values and represents the true water molecular diffusion. However, in an actual environment, 
proton pools cannot be divided completely, and so the mono- and bi-exponential models can be confounded by a mismatch from the heterogeneous nature of tissues. Therefore, the stretched exponential model was introduced recently to overcome the limitations of previous models (30). It measures the heterogeneity of the diffusion in an environment by measuring the degree of deviation from the mono-exponential behavior $(31,32)$. This model is based on the theory that protons show a continuous distribution of diffusions at different diffusion rates without an assumption of tissue compartmentalization (30). From this model, the heterogeneity index $(\alpha)$ and distributed diffusion coefficient (DDC) can be measured. Heterogeneity index $(\alpha)$, which presents values ranging from 0 to 1 , represents the deviation of signal intensities from the mono-exponential curve (33). A high $\alpha$ value close to 1 indicates low intravoxel diffusion heterogeneity by approaching monoexponential decay (30). The DDC represents the mean intravoxel diffusion rate in the presence of heterogeneity.

DWI can be applied in the evaluation of both focal liver lesion and diffuse liver disease in children $(26,34)$. The stretched exponential model is an emerging technique, and only a few studies have utilized this model to date for pediatric imaging, such as in the context of brain tumors or body oncology $(35,36)$. In adults, the stretched exponential model showed higher diagnostic performance for determining significant hepatic fibrosis than did the mono-exponential model (32).

\section{MR Elastography}

MR elastography assesses tissue stiffness by measuring the speed of shear waves propagating within it. When performing MR elastography, special hardware is required. The active driver, which is similar to an audio device, is located outside the scanner room. It delivers vibrational energy to the passive driver $(60 \mathrm{~Hz}$ for liver MR elastography) through the connecting tube. The passive driver is located near the right anterior abdominal wall and delivers vibrations into the liver. The passive driver must be in contact with the body, and a folded towel is occasionally placed between the driver and child to improve mechanical coupling between the body wall and the driver (37). The driver power is reduced by $20 \%$ in pediatric patients (aged 5-18 years) and by $40 \%$ to $50 \%$ in very young children (younger than two years of age) versus the level in typical adult patients in order to prevent theoretical injuries. For patients aged between two and five, an appropriate intermediate power level can be subjectively selected based on the patient's height, weight, and size (38).

The MR elastography technique can be implemented on conventional 1.5T or 3T scanners, and the measured mechanical properties do not depend on magnetic field strength, since the measured stiffness is contingent upon frequency (39). The response of the tissue to the mechanical stress generated by the vibrations is measured using standard MR phase-contrast imaging sequences with the addition of motion-encoding gradients synchronized with the vibration input. At 1.5T, a 2D GRE sequence is often used, while at $3 \mathrm{~T}$, either a $2 \mathrm{D}$ GRE or $2 \mathrm{D}$ spin-echo echo-planar imaging (EPI) sequence is generally used. Spinecho EPI MR elastography sequences have been reported to demonstrate fewer artifacts and to generate larger regions of interest in comparison with 2D GRE imaging (40, 41). These sequences produce wave images, which allow the visualization of the propagating shear waves within the target tissue. Inversion algorithms are applied to the acquired wave images and quantitative maps of tissue stiffness are generated, so-called elastograms (42). Regions of interest are selected, avoiding large blood vessels, to provide an overall estimate of parenchymal stiffness in kPa. MR elastography should ideally be performed after 4-6 hours of fasting. Postprandial status does not significantly change the stiffness of the normal liver, but may cause increased liver stiffness in chronic liver disease because of the transient increase in portal venous flow after meals (43).

MR elastography has been proven to be an accurate method for diagnosing and staging liver fibrosis, albeit mostly in adults (44). In comparison with other methods, liver MR elastography covers much larger volumes of the liver cross-sectionally and provides a global noninvasive assessment of liver parenchyma. Liver MR elastography can be performed in most patients, including those with obesity, anatomical variations, and substantial ascites (37). MR elastography is associated with a high rate of successful examinations in clinical practice, including with a more than $96 \%$ success rate in pediatric and young adult patients (45). MR elastography also shows good reproducibility and repeatability with high reliability across vendor platforms, field strengths, and pulse sequences, with an overall intraclass correlation coefficient of more than 0.68 (46).

\section{Dynamic Contrast-Enhanced MRI}

DCE MRI involves continuous measurement of the T1 signal intensity of the region before, during, and after the 
contrast injection (47). For the acquisition of DCE MRI images, three steps are performed as follows: 1 ) baseline T1 mapping, 2) dynamic data acquisition, and 3) arterial input function (AIF) assessment $(47,48)$. For T1 mapping, a fixed $\mathrm{T} 1$ value or measured $\mathrm{T} 1$ value obtained from variable flip angles, inversion recovery, or the look-locker technique can be used (47). For dynamic data acquisition, short temporal resolutions (about 2-4 seconds) and an adequate spatial resolution (in-plane resolution of 0.5-1.7 $\mathrm{mm}$ ) are required for quantification (47). After quantification, measurement of AIF is performed to identify the contrast concentration gradient between the intravascular space and extravascular extracellular space (EES) to minimize inflow effects from large vessels (49). Using a semiquantitative model-free method, parameters such as the area under the curve and maximum or relative signal intensities can be quantified from the slope $(47,50)$. Quantitative analysis from a pharmacokinetic model, such as the extended Toft's model, has recently been adopted and yields more physiologic, accurate, and reproducible results in comparison with those obtained using the model-free method (48). The extended Toft's model is based on the two-compartment theory, considering the idea that contrast can move between the intravascular plasma space and EES. Using this model, the volume of the plasma compartment $(V p)$, volume of the extracellular compartment (Ve), volume transfer constant between the plasma and EES $\left(\mathrm{K}^{\mathrm{trans}}\right)$, and rate constant $\left(\mathrm{K}_{\mathrm{ep}}\right.$ $\left.=K^{\text {trans }} / \mathrm{Ve}\right)$ can be measured $(47,50)$.

Several specific considerations are needed in liver DCE MRI (51), because the liver receives a unique dual supply of blood from the portal vein and hepatic artery. Moreover, the contrast agent can freely pass through plasma and extracellular spaces because of the highly fenestrated endothelial cells in the liver. Previous studies have applied different models for analyzing DCE MRI findings in the liver, but a single method has not been standardized yet for liver application. A dual-input single-compartment model was applied, but it could not reflect the relationship between plasma space and EES (51). A few previous studies have also considered a dual-input two-compartment model using software $(52,53)$, but no software considering this model is available at this time from major vendors. Therefore, as an alternative method, a single-input two-compartment model was used in recent investigations by considering that the abdominal aorta could be used as a surrogate input source of the liver $(51,54)$.

No study to date has assessed the utility of liver DCE
MRI in the pediatric population. This technique is more in the realm of research rather than a clinically accepted technique and the post-processing is not widely available. The performance of DCE MRI is still challenging in the liver and also in children, because accurate T1 mapping, high temporal resolution, the selection of an adequate kinetic model, and reliable reproducible AIF acquisition are necessary. Therefore, the standardization of imaging acquisition and data analysis methods is crucial for stable utilization of DCE MRI in the liver and in pediatric patients.

CT

With the advances in CT technology, DECT can be used for material differentiation and contrast identification. DECT-based multi-material decomposition algorithms generated fat volume fraction images, enabling accurate and reproducible liver fat quantification in an adult study (55). DECT could thus be a less-invasive technique for the assessment of hepatic fat content. However, the radiation exposure associated with $\mathrm{CT}$ is still a major limitation in its use in children.

In 2016, Siegel et al. (56) demonstrated reduced radiation exposure in DECT in comparison with estimated singleenergy $\mathrm{CT}$ levels in children while maintaining a similar contrast profile and contrast-to-noise ratio. Therefore, if used as an optimized study method in appropriate indications, DECT could help in diagnosing liver diseases in children.

\section{Clinical Applications of Quantitative Hepatobiliary Imaging}

\section{Biliary Atresia}

Biliary atresia is a perinatal disease of unclear etiology characterized by inflammatory obstruction of the biliary tress, leading to biliary cirrhosis. US elastography, especially SWE, have been used in the diagnosis of biliary atresia patients since liver SWE values are higher in biliary atresia patients compared to those in other infantile liver diseases $(57,58)$. However, one study showed that its diagnostic performance does not exceed that of grayscale US (59). When using MRI, the T2 relaxation time was not different in patients with and without biliary atresia and did not correlate with the stage of hepatic fibrosis in infants with cholestasis (60). However, liver ADC values in biliary atresia patients were lower than those in a control group and had 
a negative correlation with the degree of liver fibrosis following Kasai operation $(61,62)$. On IVIM assessments, hepatic $D^{*}$ values were lower than those in normal children and showed negative correlation with increased liver stiffness (63). Figure 3 shows the utility of mon-, bi-, and stretched exponential models in the liver of a biliary atresia patient. However, there is no published study evaluating the usefulness of bi- or stretched exponential models for the diagnosis and follow-up of biliary atresia patients yet.

\section{Hepatic Fibrosis}

US elastography, especially SWE, allows accurate assessment of liver fibrosis with a high success rate, even in children with early stage fibrosis (64). US elastography also showed good performance in diagnosing portal hypertension in children in a systematic review and meta-analysis (65). The summary sensitivity was 90\% (95\% CI: 83-94\%), while specificity was 79\% (95\% CI: 73-84\%). Another study performed only on the subjects of transient elastography also showed good diagnostic performance, with a sensitivity of $95 \%$ (95\% CI: $74-99 \%)$ and a specificity of $90 \%(95 \%$ CI: $81-95 \%$ ) for the diagnosis of significant liver fibrosis (stage $\geq$ F2) in children (66). However, the cutoff values for differentiating each fibrosis stage and for diagnosing portal hypertension varied greatly $(65,66)$. Optimal cutoff values according to the specific cause of liver disease are yet to be established. As an alternative, additional spleen SWE measurements could be helpful in differentiating portal

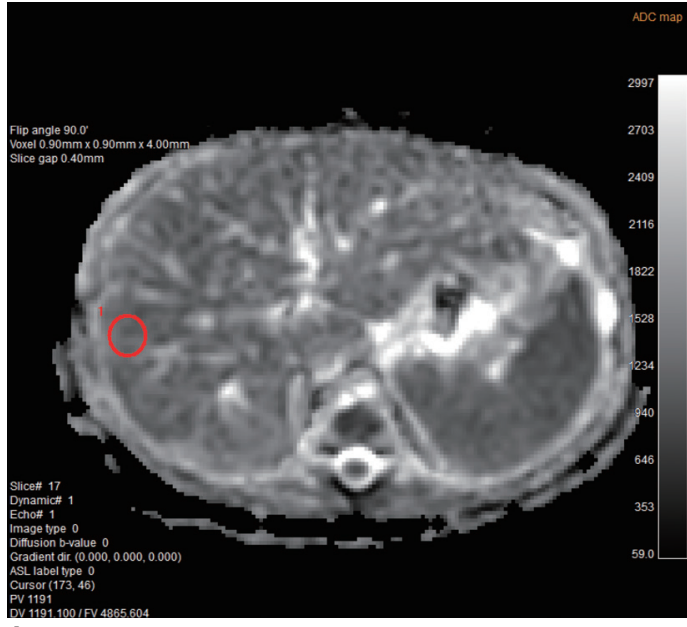

A

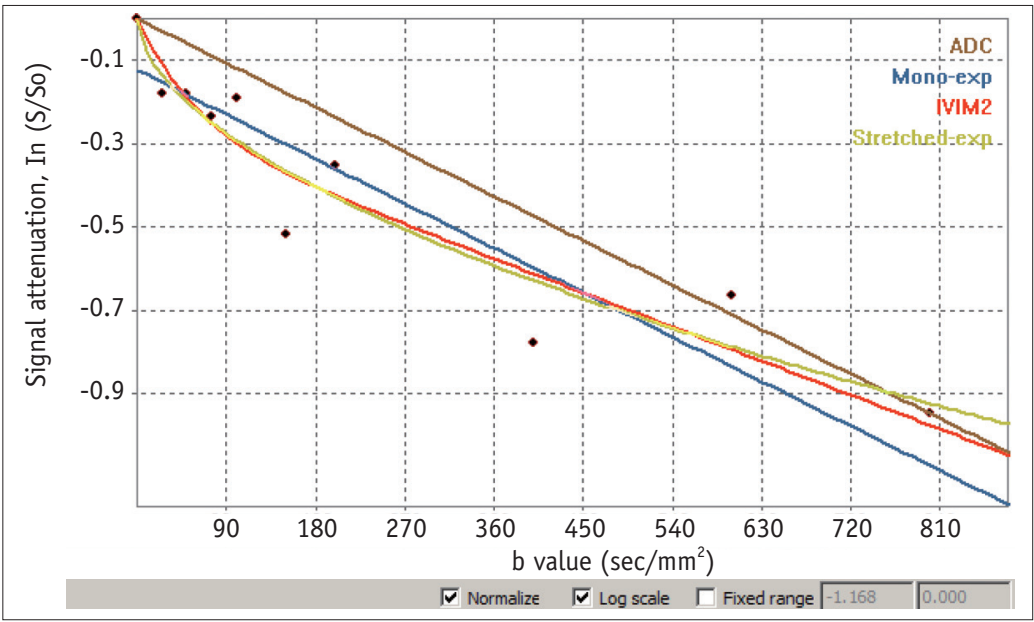

B

Fig. 3. 2-month-old girl with biliary atresia.

A. Liver MRI with mono-, bi-, and stretched exponential model DWI shows red circular ROI in liver parenchyma. B. Curves can be obtained according to models. From these models, ADC value was $1.2 \times 10^{-3} \mathrm{~mm}^{2} / \mathrm{s}, \mathrm{D}^{*}$ value was $19.6 \times 10^{-3} \mathrm{~mm}^{2} / \mathrm{s}, \mathrm{f}$ value was $0.2, \mathrm{D}$ was $0.9 \times 10^{-3} \mathrm{~mm}^{2} / \mathrm{s}$, distributed diffusion coefficient was $1.1 \times 10^{-3} \mathrm{~mm}^{2} / \mathrm{s}$, and $\alpha$ value was $0.5 . A D C=$ apparent diffusion coefficient, $\mathrm{D}=$ true diffusion, $\mathrm{D}^{*}=$ pseudo-diffusion, DWI $=$ diffusion-weighted imaging, $\mathrm{f}=$ perfusion fraction, $\alpha=$ heterogeneity index

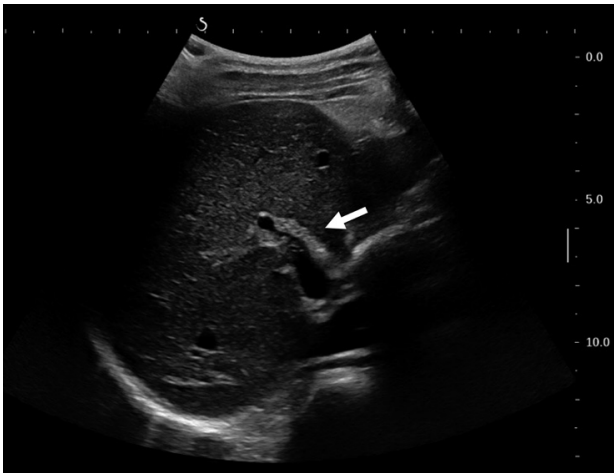

A

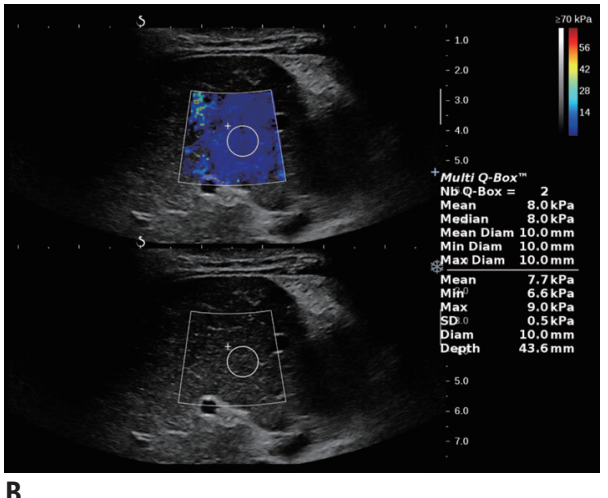

B

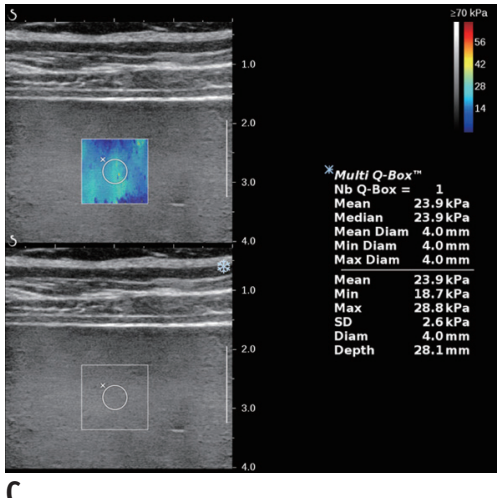

C

Fig. 4. 12-year-old girl with liver fibrosis after Kasai operation for biliary atresia.

A. Liver shows periportal hyperechogenicity (arrow) with left lobe atrophic change and lobulated contour. B. Shear wave elastography image of liver shows blue color in acquisition box with measured mean elasticity value of $8.0 \mathrm{kPa}$. Value in transient elastography was $7.7 \mathrm{kPa}$ (not shown). C. Patient showed splenomegaly considering her age (more than $11.6 \mathrm{~cm}$, not shown), and spleen shear wave elastography value was $23.9 \mathrm{kPa}$. 
hypertension in children (Fig. 4) (67). The cutoff value of splenic point SWE for portal hypertension was $3.14 \mathrm{~m} / \mathrm{s}$, with a specificity of $98.6 \%$ and a sensitivity of $68.2 \%$, even though the diagnostic performance was lower than that for the liver.

One meta-analysis in the adult population had reported the high diagnostic performance of liver MR elastography in staging fibrosis, with an area under the receiver operating characteristic curve of more than 0.88 in patients with significant fibrosis ( $\geq$ stage 2 ), regardless of body mass index and the etiology of chronic liver disease (68). Another adult meta-analysis reported that a measured change of $22 \%$ or greater in hepatic stiffness indicated that a true change in stiffness occurred over time with $95 \%$ confidence (Fig. 5) (44). MR elastography also has been used in the pediatric population. Xanthakos et al. (69) evaluated 35 patients aged 4-20 years with chronic liver disease, mainly nonalcoholic fatty liver disease (NAFLD), and suggested a cutoff value of $2.71 \mathrm{kPa}$ for significant fibrosis (stages 2-4).
There was no difference between liver MR elastography values in patients with and without portal hypertension or gastroesophageal varices, even though spleen MR elastography values were higher with a cutoff value of 9.9 $\mathrm{kPa}$ for predicting gastroesophageal varices (Fig. 6) (70). Since there may be confounding factors related to the liver parenchyma in these patients, such as inflammation, the presence of these confounding factors should always be considered prior to interpreting liver MR elastography values.

\section{Nonalcoholic Fatty Liver Disease}

NAFLD is a condition that is characterized by the presence of $>5 \%$ fat in hepatocytes and is an increasing cause of chronic liver disease in children. If adequate and early precautions are not taken, it can progress to cirrhosis. Quantitative ultrasonographic techniques have been developed to evaluate hepatic fat, including speed of sound estimation, backscatter coefficient, shear wave dispersion,
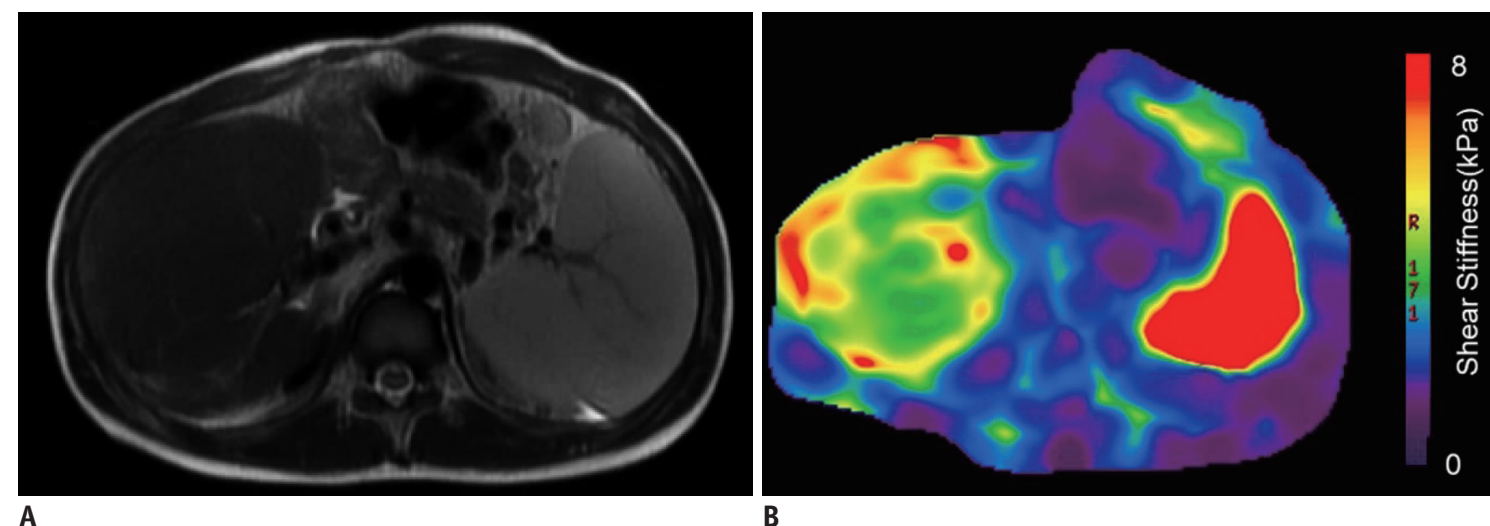

A

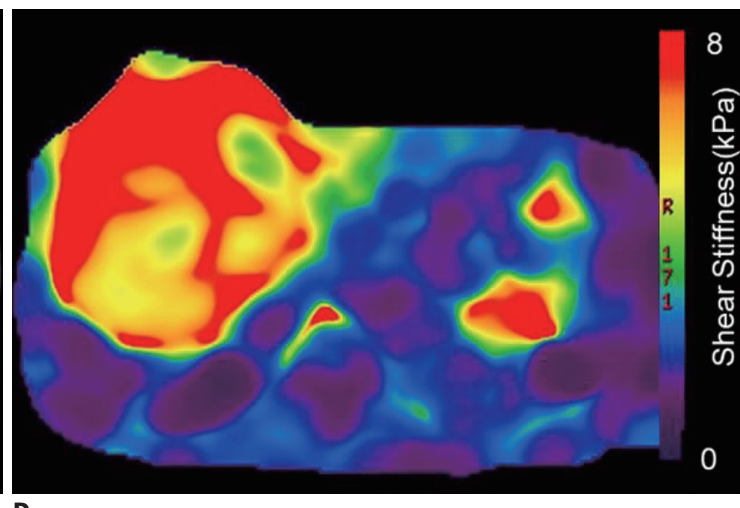

C

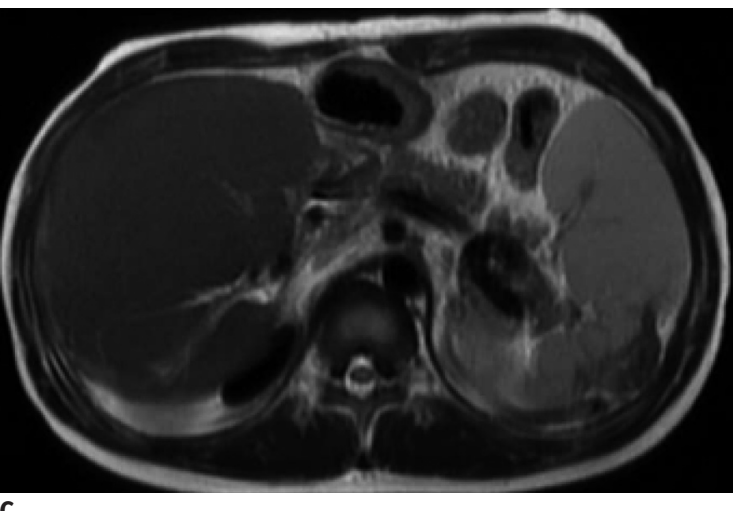

D

Fig. 5. 9-year-old girl with biliary atresia after Kasai operation and grade 2 gastroesophageal varices.

A. Axial T2-weighted imaging of liver shows hypertrophied right lobe and atrophic left lobe of liver with splenomegaly and engorged portal collateral vessels. B. Color map in MR elastography shows green to red color of liver, indicating increased stiffness. Measured mean liver elasticity was $4.1 \mathrm{kPa}$. C. Two years later, axial T2-weighted MR image shows aggravated liver cirrhosis with partial splenic artery embolization status in this patient. D. On follow-up MR elastography imaging, liver stiffness further increased, with mean value of $6.9 \mathrm{kPa}$. This patient underwent liver transplantation 2 months after last exam. 
and attenuation parameters (2). A recent study suggested simple grayscale US quantification of a hepatorenal ratio, which was obtained by dividing the mean liver by the mean kidney values using a readily available picture archiving and communication system (Fig. 7) (71). This parameter showed $88.2 \%$ sensitivity and $91.4 \%$ specificity for predicting hepatic steatosis when using the cutoff value of 1.5 in children. However, other pathologic conditions such as fibrosis and inflammation may intensify liver echogenicity and can result in misinterpretations in this context.

PDFF is known to have high correlation with the histologic grades of hepatic steatosis in adults $(r=0.69-$ $0.74)$ and also in children $(r=0.73)(2,72)$. PDFF has also shown a close agreement with MR spectroscopy values in children (73). Moreover, the radial sampling technique provides an accurate measurement of PDFF in free-breathing status by showing mean differences of less than $0.7 \%$ in comparison with PDFF in breath-holding status in children (74). In addition to the diagnosis of hepatic steatosis, PDFF can be used as a tool for the follow-up of hepatic steatosis in children (Fig. 8) (75).

NAFLD patients may show coexisting hepatic iron overload and fat accumulation, and this feature is known to accelerate liver injury (76). In these cases, both detection and quantification of iron and fat can become difficult, especially with conventional MRI. However, the quantification of both iron and fat is still feasible using recent methods that can calculate R2* and PDFF simultaneously (77). This approach can help clarify the coexistence of siderosis and steatosis. In such cases, it is essential to use the multi-peak fat model for accurate quantification of fat (78).

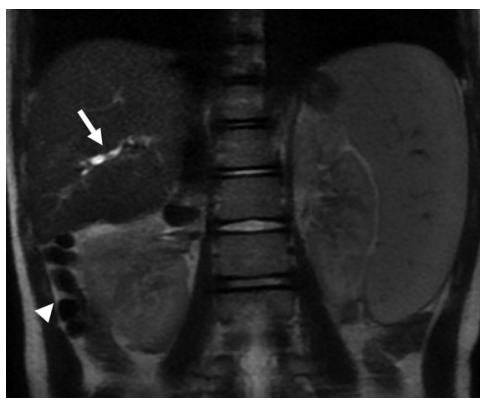

A

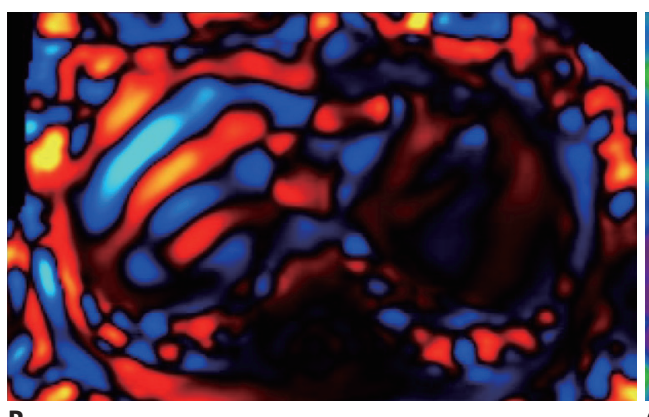

B

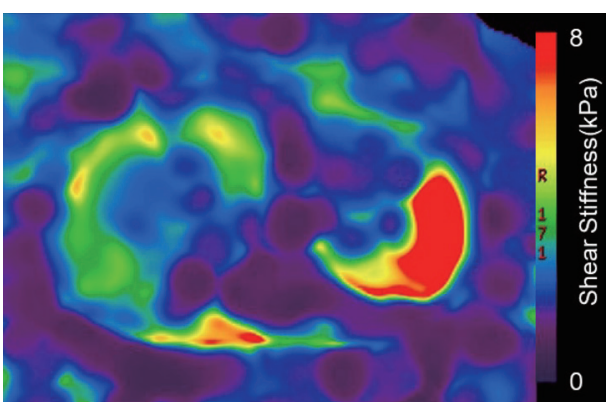

Fig. 6. 9-year-old boy with biliary atresia and previous Kasai operation.

A. Coronal single-shot fast spin-echo T2-weighted image shows multiple tiny intrahepatic biliary cysts (arrow) in liver and splenomegaly. Patient also showed engorged mesenteric veins (arrowhead) at right subhepatic area, suggesting portosystemic collateral pathways. B. MR elastography wave image shows thick blue and red waves within liver, consistent with elevated shear wave speed (increased wavelength), reflective of liver stiffness. C. MR elastogram color map shows blue to green color of liver and red color of spleen. Measured hepatic stiffness was $3.2 \mathrm{kPa}$ and spleen stiffness was $10.7 \mathrm{kPa}$.

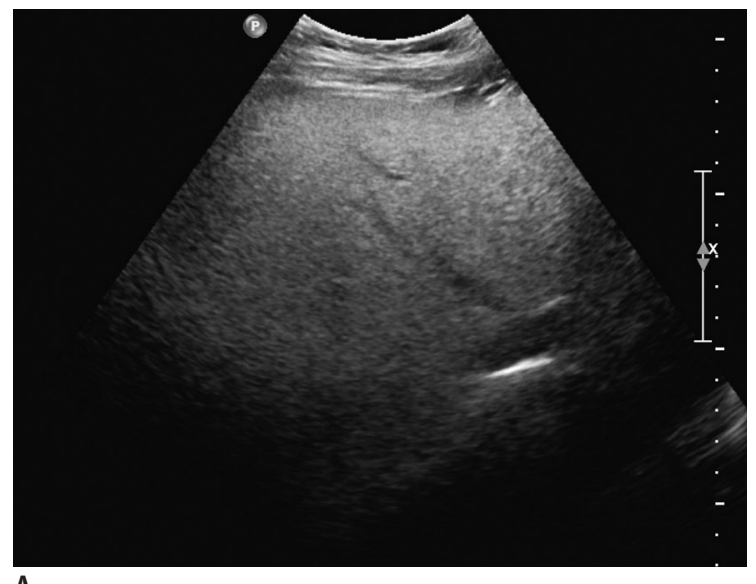

A

Fig. 7. 10-year-old boy with fatty liver.

A. Grayscale image obtained with liver ultrasonography demonstrates hepatomegaly with diffusely increased liver parenchymal echo, indistinct hepatic veins, and poor diaphragm visualization. B. Longitudinal scan of liver including right kidney shows echo difference and hepatorenal ratio of 2.3, which is above cutoff value of 1.5 in children. On liver MRI, hepatic fat fraction was $32 \%$. 
In recent studies with children, liver steatosis and fibrosis showed independent effects on DWI. Bi-exponential model DWI (IVIM) revealed that microvascular perfusion and true water molecular diffusion affected liver steatosis and fibrosis differently $(63,79)$. Hepatic fat and blood volume measured by $f$ value showed a positive correlation, while hepatic fibrosis and endovascular blood flow velocity measured by $D^{*}$ showed a negative correlation (63).

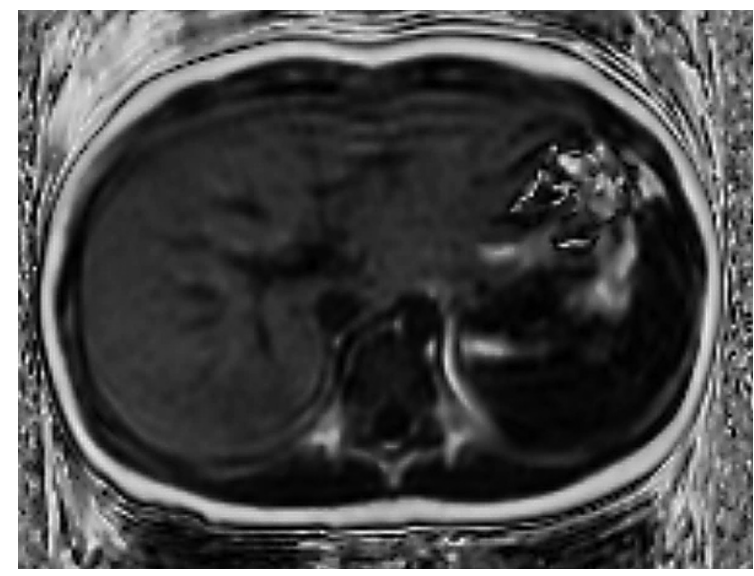

A

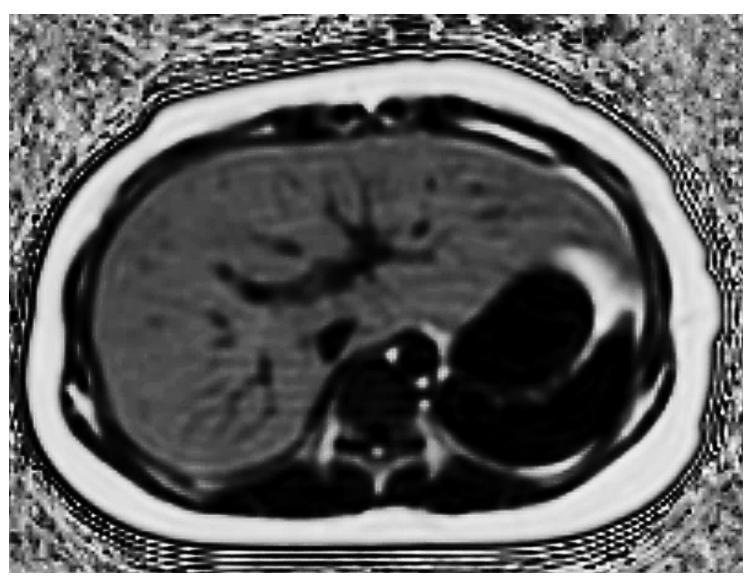

B

Fig. 8. 11-year-old boy with hepatic steatosis.

MR proton density fat fraction of liver was $31 \%$ when patient was 6 years old (A) and increased to $51 \%, 5$ years later (B).

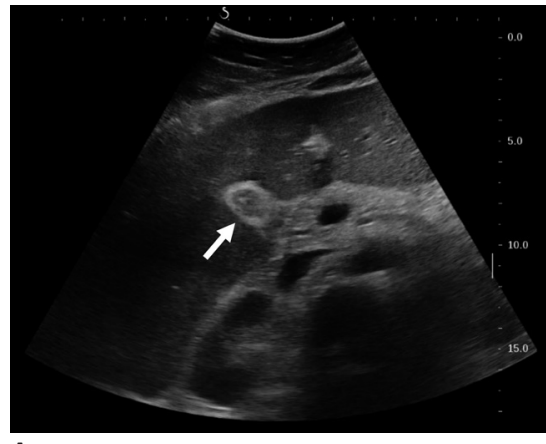

A

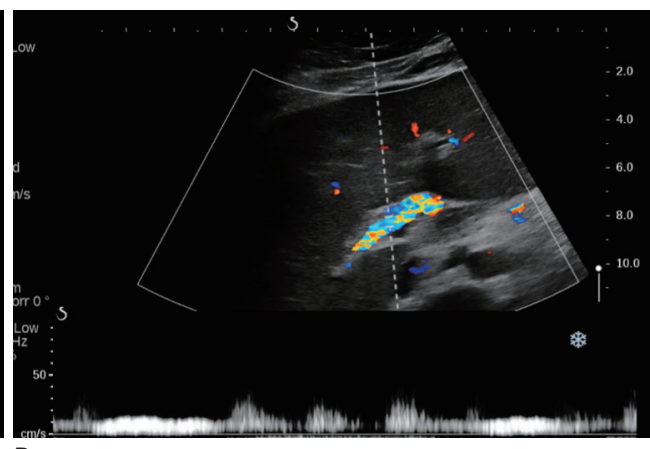

B

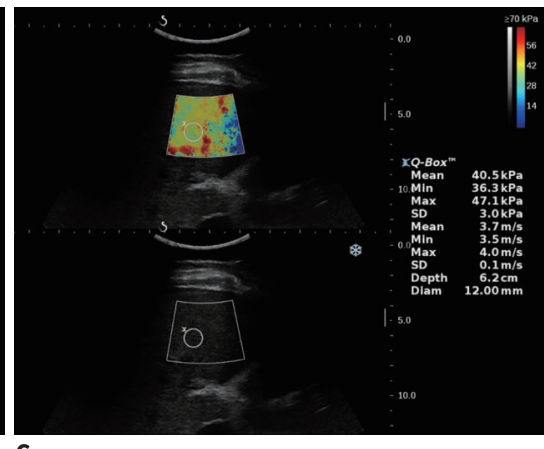

C

Fig. 9. 13-year-old girl with sinusoidal obstruction syndrome or veno-occlusive disease.

A. Transverse ultrasound image of liver shows hepatomegaly with periportal edema and collapsed gallbladder wall edema (arrow). There was moderate amount of ascites (not shown). B. Doppler image of liver hilum shows decreased portal vein flow. C. Shear wave elastography image of liver shows yellow to red color in acquisition box with measured mean elasticity value of $40.5 \mathrm{kPa}$.

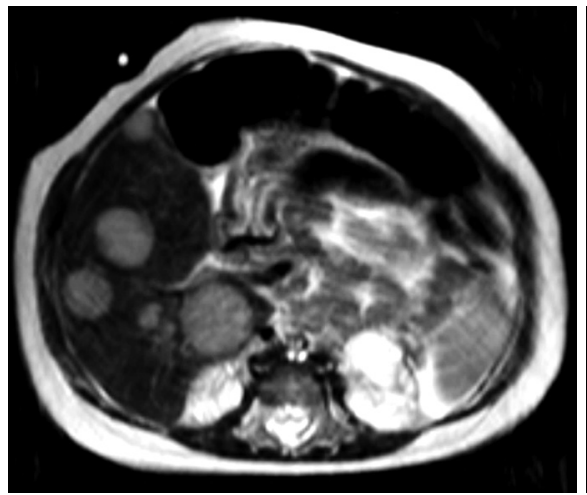

A

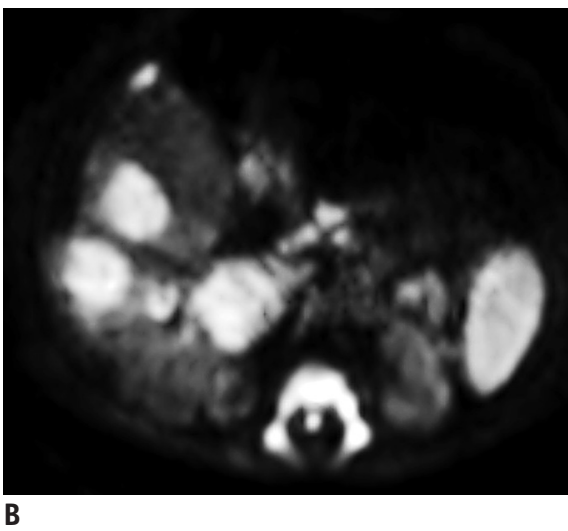

B

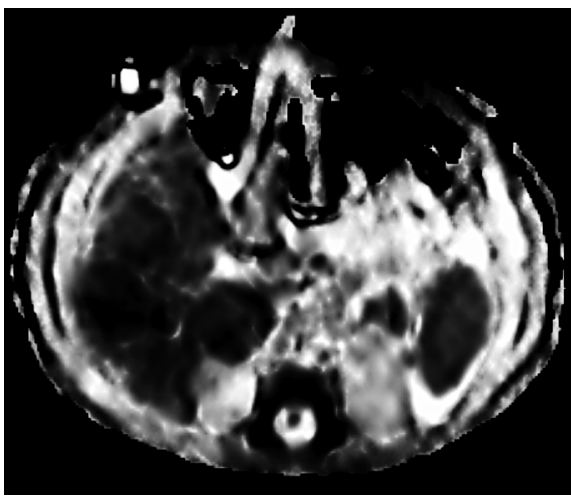

C

Fig. 10. 4-month-old girl with hepatic masses.

A. Multiple high signal intensity masses are noted in liver on T2-weighted image. B. On DWI, masses show high signal intensity on high b-value. C. On ADC map, masses show low ADC value, indicating that they are malignant. Masses were confirmed as hepatoblastoma. 

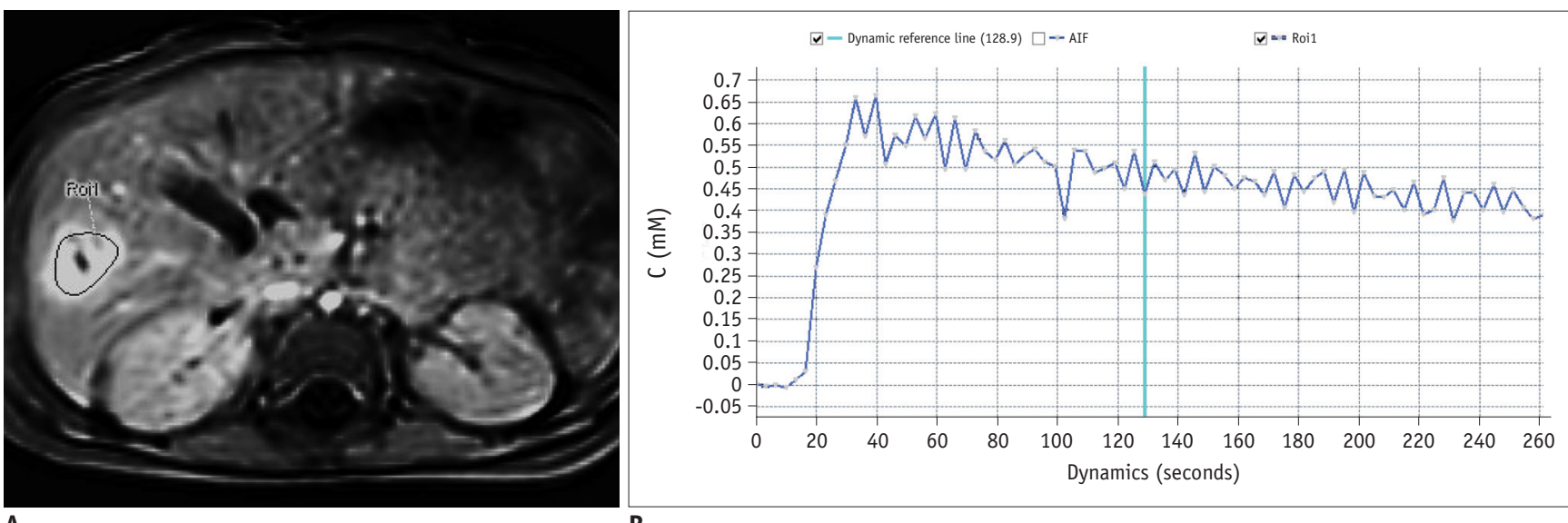

A

B

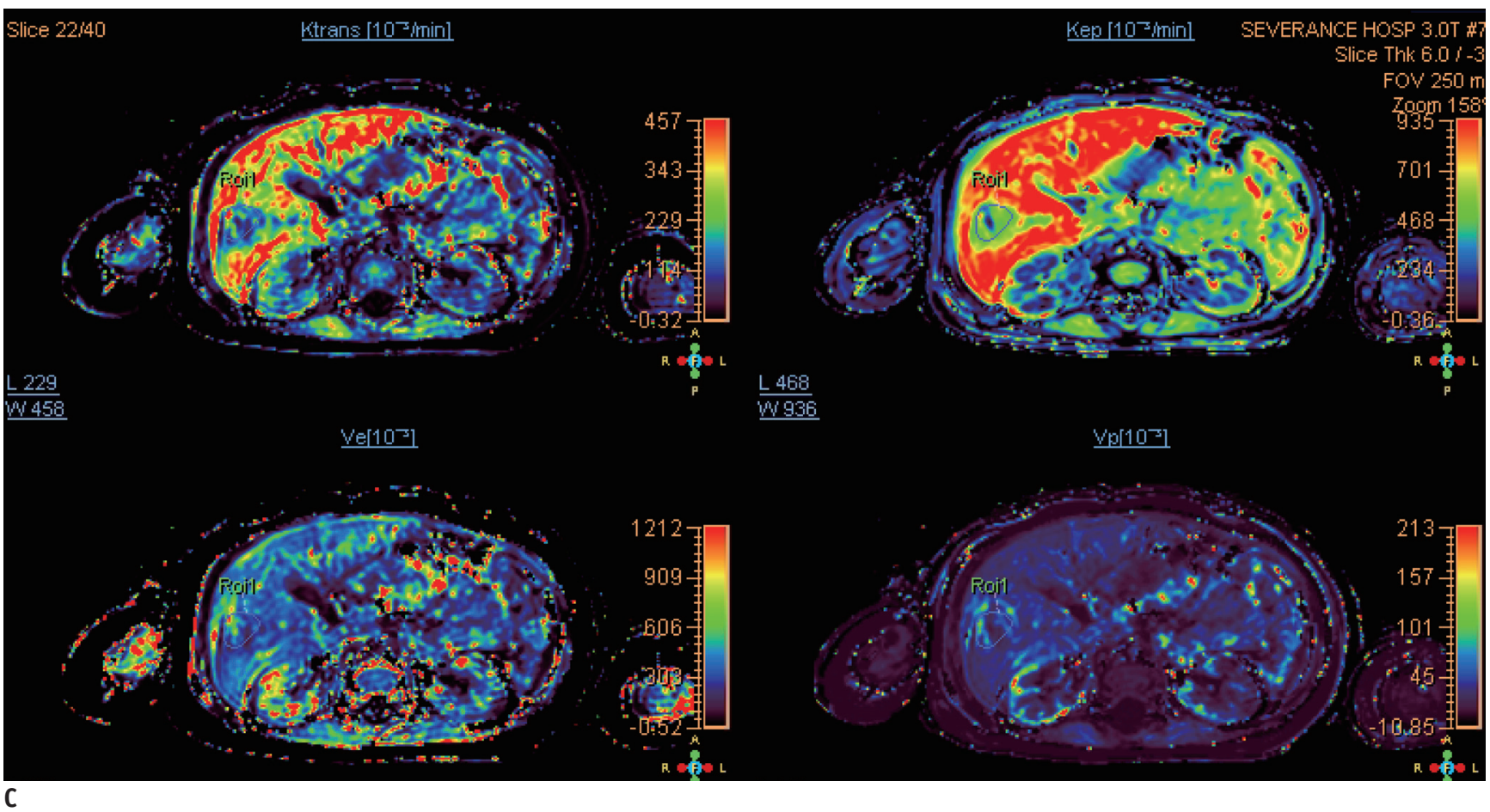

Fig. 11. 3-month-old boy with liver hemangioma.

A. DCE MRI of liver shows enhancing mass lesions in right lobe with central non-enhancing portion. B. When drawing ROI in mass lesion as in (A), slope showing early arterial and persistent enhancement was obtained. C. Values obtained from multi-parametric maps were as follows: $\mathrm{K}^{\text {trans }}=$ $174.3 \times 10^{-3} / \mathrm{min}, \mathrm{Kep}=490.9 \times 10^{-3} / \mathrm{min}, \mathrm{Ve}=355.1 \times 10^{-3}$, and $\mathrm{Vp}=41.6 \times 10^{-3}$. AIF $=$ arterial input function, DCE $=$ dynamic contrast-enhanced, $\mathrm{Kep}=$ rate constant $\left(\mathrm{K}^{\text {trans }} / \mathrm{Ve}\right), \mathrm{K}^{\text {trans }}=$ volume transfer constant between plasma and extravascular extracellular space, $\mathrm{Ve}=$ volume of extracellular compartment, $\mathrm{Vp}=$ volume of plasma compartment

These findings are different from those obtained with MR elastography in NAFLD patients. MR elastography performs significantly better in distinguishing stages 0 and 1 versus stage 2 or higher fibrosis in patients without steatosis than in those with steatosis (80). This suggests a confounding effect of steatosis or inflammation in assessments of fibrosis in the population with NAFLD. Moreover, there was a lack of agreement between MR elastography stiffness values and histopathological scores in the presence of moderate to high liver fat in pediatric and young adults (81).

A few recent trials used DCE MRI in animal models for diffuse liver disease, including one that found increased $\mathrm{K}^{\text {trans }}$ in hepatic steatosis and fibrosis in NAFLD (52). However, in another study using a rat liver fibrosis model without the effect of steatosis, $\mathrm{K}^{\text {trans }}$ was negatively correlated with fibrosis stages (53). The utility of DCE MRI in evaluating diffuse hepatic disease in children needs further research. 

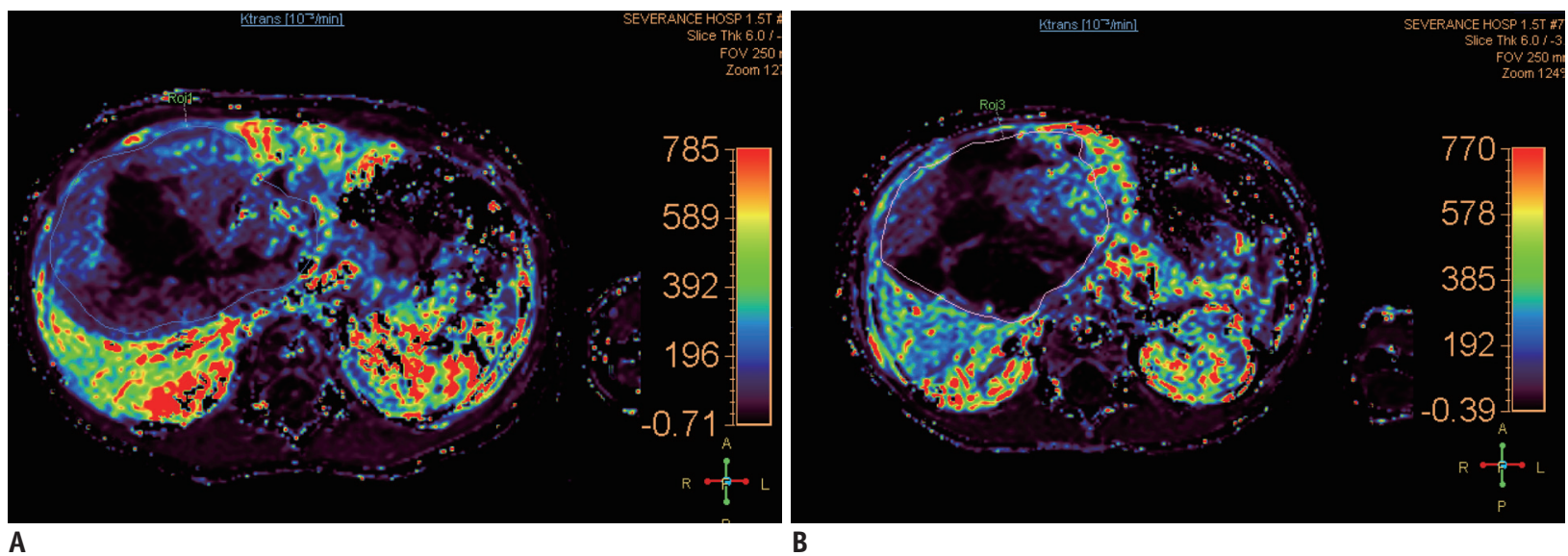

Fig. 12. 4-year-old girl with hepatoblastoma.

A. At initial liver DCE MRI of hepatoblastoma, $\mathrm{K}^{\text {trans }}$ was $86.7 \times 10^{-3} / \mathrm{min}$. B. Follow-up DCE MRI shows treatment response. At 3 months after chemotherapy, tumor size had decreased and $\mathrm{K}^{\text {trans }}$ had also decreased to $70.6 \times 10^{-3} / \mathrm{min}$.

\section{Hepatic Veno-0cclusive Disease}

Sinusoidal obstruction syndrome (SOS) or hepatic venoocclusive disease (VOD) is a complication that occurs after hematopoietic cell transplantation. It occurs more commonly in children (up to $20 \%$ ) and demonstrates a high mortality rate (5). Therefore, accurate and early diagnosis of SOS/VOD is clinically important. Even though the diagnosis of SOS/VOD is based on clinical criteria, US imaging findings of both grayscale and Doppler criteria linked to portal hypertension are important in order to successfully differentiate other conditions. Here, the combination of hepatomegaly, ascites, reduced flow in the portal vein measured in the intrahepatic portal vein (threshold of 10 $\mathrm{cm} / \mathrm{s}$ ), elevated resistive index $(>0.8)$ in the hepatic artery, and reduced but monophasic flow in the right hepatic vein (measured $>80 \mathrm{~mm}$ from the inferior vena cava) seem to be the most promising imaging features (Fig. 9) (5). Even though SMI successfully detected differences in flow grades between normal and undescended testes in young children better than conventional power Doppler imaging (4), little is known regarding the utility of SMI for assessing pediatric liver disease at present.

A combination of Doppler US with SWE could assist in the diagnosis and follow-up of SOS/VOD (Fig. 9) (5). Liver shear wave velocity was elevated in an SOS/VOD animal model in proportion to the degree of sinusoidal injury and lobular inflammation (82). This finding might be more sensitive than the conventional Doppler criteria of larger hepatic vessels for the early diagnosis of SOS/VOD. Another study showed that time-intensity curve analysis could provide a more objective approach in the early diagnosis of SOS/VOD
(83). CEUS might best reflect pathophysiological changes in SOS/VOD (5).

\section{Hepatic Mass}

Previous reports suggested that SWE may help in differentiating malignant from benign focal liver lesions with good intra-observer reproducibility in the adult population $(84,85)$. However, SWE has limited application in assessments of pediatric focal liver lesion.

The use of the mono-exponential model DWI is better than T2-weighted images for the detection of focal liver lesions and facilitates differentiation of benign and malignant lesions $(26,86)$. In neonates and young infants, hepatic lesions with decreased signal intensity on an ADC map could lead to the diagnosis of hepatoblastoma (Fig. 10) (87). In addition, the differentiation of hepatic adenoma or hepatocellular carcinoma could be aided by DWI as it is in adults (86).

Using DCE MRI in an animal model for the evaluation of liver lesion, $\mathrm{K}^{\text {trans }}$ was shown to increase in hepatocellular carcinoma (88). Our institution uses pediatric liver DCE MRI at $1.5 \mathrm{~T}$ with a 3D turbo field echo sequence and a temporal resolution of one dynamic per 4 to 5 seconds and 60 dynamics (at least 20 dynamics are required for the analysis). As in our cases, liver DCE MRI could be used for the quantification of liver lesions (Fig. 11) and for the monitoring of treatment response in hepatic tumors (Fig. 12) in serial standardized protocols. However, further validation using this technique is still needed. 


\section{CONCLUSION}

At this time, we can use variable quantitative imaging techniques to better understand hepatobiliary diseases in children. Specifically, not only MRI but also US can be used to evaluate changes in tissue components, vascularity, and elasticity. We can use these techniques for evaluation of biliary atresia, hepatic fibrosis, NAFLD, SOS/VOD, and hepatic masses in children. Due to the risk of radiation, the effectiveness of DECT for material differentiation has not yet been verified in children. With current and ongoing advances in technology, each imaging technique offers a unique advantage, and the use of the appropriate imaging modality according to a particular disease or patient condition may help to improve diagnosis and treatment by presenting more accurate and objective data in children.

\section{Conflicts of Interest}

The authors have no potential conflicts of interest to disclose.

\section{ORCID iDs}

Mi-Jung Lee

https://orcid.org/0000-0003-3244-9171

Haesung Yoon

https://orcid.org/0000-0003-0581-8656

Hyun Joo Shin

https://orcid.org/0000-0002-7462-2609

Myung-Joon Kim

https://orcid.org/0000-0002-4608-0275

\section{REFERENCES}

1. Gubernick JA, Rosenberg HK, Ilaslan H, Kessler A. US approach to jaundice in infants and children. Radiographics 2000;20:173-195

2. Ozturk A, Grajo JR, Gee MS, Benjamin A, Zubajlo RE, Thomenius KE, et al. Quantitative hepatic fat quantification in non-alcoholic fatty liver disease using ultrasound-based techniques: a review of literature and their diagnostic performance. Ultrasound Med Biol 2018;44:2461-2475

3. Megremis SD, Vlachonikolis IG, Tsilimigaki AM. Spleen length in childhood with US: normal values based on age, sex, and somatometric parameters. Radiology 2004;231:129-134

4. Lee YS, Kim MJ, Han SW, Lee HS, Im YJ, Shin HJ, et al. Superb microvascular imaging for the detection of parenchymal perfusion in normal and undescended testes in young children. Eur J Radiol 2016;85:649-656

5. Dietrich CF, Trenker C, Fontanilla T, Görg C, Hausmann A, Klein
$S$, et al. New ultrasound techniques challenge the diagnosis of sinusoidal obstruction syndrome. Ultrasound Med Biol 2018;44:2171-2182

6. Chang S, Kim MJ, Kim J, Lee MJ. Variability of shear wave velocity using different frequencies in acoustic radiation force impulse (ARFI) elastography: a phantom and normal liver study. Ultraschall Med 2013;34:260-265

7. Shin HJ, Kim MJ, Kim HY, Roh YH, Lee MJ. Comparison of shear wave velocities on ultrasound elastography between different machines, transducers, and acquisition depths: a phantom study. Eur Radiol 2016;26:3361-3367

8. Shin HJ, Kim MJ, Kim HY, Roh YH, Lee MJ. Optimal acquisition number for hepatic shear wave velocity measurements in children. PLoS One 2016;11:e0168758

9. Kim YY, Kim MJ, Shin HJ, Yoon H, Kim HY, Lee MJ. Interconversion of elasticity measurements between two-dimensional shear wave elastography and transient elastography. Med Ultrason 2018;20:127-133

10. Galina P, Alexopoulou E, Zellos A, Grigoraki V, Siahanidou T, Kelekis NL, et al. Performance of two--dimensional ultrasound shear wave elastography: reference values of normal liver stiffness in children. Pediatr Radiol 2019;49:91-98

11. Lee MJ, Kim MJ, Han KH, Yoon CS. Age-related changes in liver, kidney, and spleen stiffness in healthy children measured with acoustic radiation force impulse imaging. Eur J Radiol 2013;82:e290-e294

12. Ntoulia A, Anupindi SA, Darge K, Back SJ. Applications of contrast-enhanced ultrasound in the pediatric abdomen. Abdom Radiol (NY) 2018;43:948-959

13. Sidhu PS, Cantisani V, Deganello A, Dietrich CF, Duran C, Franke $D$, et al. Role of contrast-enhanced ultrasound (CEUS) in paediatric practice: an EFSUMB position statement. Ultraschall Med 2017;38:33-43

14. Laugesen NG, Nolsoe CP, Rosenberg J. Clinical applications of contrast-enhanced ultrasound in the pediatric work-up of focal liver lesions and blunt abdominal trauma: a systematic review. Ultrasound Int Open 2017;3:E2-E7

15. Shin HJ, Chang EY, Lee HS, Hong JH, Park G, Kim HG, et al. Contrast-enhanced ultrasonography for the evaluation of liver fibrosis after biliary obstruction. World J Gastroenterol 2015;21:2614-2621

16. Towbin AJ, Serai SD, Podberesky DJ. Magnetic resonance imaging of the pediatric liver: imaging of steatosis, iron deposition, and fibrosis. Magn Reson Imaging Clin N Am 2013;21:669-680

17. Ma X, Holalkere NS, Kambadakone RA, Mino-Kenudson M, Hahn PF, Sahani DV. Imaging-based quantification of hepatic fat: methods and clinical applications. Radiographics 2009;29:1253-1277

18. Shin HJ, Kim HG, Kim MJ, Koh H, Kim HY, Roh YH, et al. Normal range of hepatic fat fraction on dual- and triple-echo fat quantification MR in children. PLoS One 2015;10:e0117480

19. Wood JC. Magnetic resonance imaging measurement of iron overload. Curr Opin Hematol 2007;14:183-190 
20. Wood JC, Ghugre N. Magnetic resonance imaging assessment of excess iron in thalassemia, sickle cell disease and other iron overload diseases. Hemoglobin 2008;32:85-96

21. McCarville MB, Hillenbrand CM, Loeffler RB, Smeltzer MP, Song $\mathrm{R}$, Li CS, et al. Comparison of whole liver and small region-ofinterest measurements of MRI liver R2* in children with iron overload. Pediatr Radiol 2010;40:1360-1367

22. Sirlin CB, Reeder SB. Magnetic resonance imaging quantification of liver iron. Magn Reson Imaging Clin N Am 2010;18:359-381, ix

23. Henninger B, Kremser C, Rauch S, Eder R, Zoller H, Finkenstedt $A$, et al. Evaluation of MR imaging with $T 1$ and $T 2 *$ mapping for the determination of hepatic iron overload. Eur Radiol 2012;22:2478-2486

24. Labranche R, Gilbert G, Cerny M, Vu KN, Soulières D, Olivié D, et al. Liver iron quantification with MR imaging: a primer for radiologists. Radiographics 2018;38:392-412

25. Storey P, Thompson AA, Carqueville CL, Wood JC, de Freitas RA, Rigsby CK. R2* imaging of transfusional iron burden at $3 \mathrm{~T}$ and comparison with 1.5T. J Magn Reson Imaging 2007;25:540-547

26. Chavhan GB, Alsabban Z, Babyn PS. Diffusion-weighted imaging in pediatric body MR imaging: principles, technique, and emerging applications. Radiographics 2014;34:E73-E88

27. Malayeri AA, El Khouli RH, Zaheer A, Jacobs MA, CoronaVillalobos CP, Kamel IR, et al. Principles and applications of diffusion-weighted imaging in cancer detection, staging, and treatment follow-up. Radiographics 2011;31:1773-1791

28. Courtier J, Rao AG, Anupindi SA. Advanced imaging techniques in pediatric body MRI. Pediatr Radiol 2017;47:522533

29. Kanematsu M, Goshima S, Watanabe H, Kondo H, Kawada $H$, Noda $Y$, et al. Diffusion/perfusion MR imaging of the liver: practice, challenges, and future. Magn Reson Med Sci 2012;11:151-161

30. Bennett KM, Schmainda KM, Bennett RT, Rowe DB, Lu H, Hyde JS. Characterization of continuously distributed cortical water diffusion rates with a stretched-exponential model. Magn Reson Med 2003;50:727-734

31. Mazaheri Y, Hötker AM, Shukla-Dave A, Akin 0, Hricak H. Model selection for high b-value diffusion-weighted MRI of the prostate. Magn Reson Imaging 2018;46:21-27

32. Seo N, Chung YE, Park YN, Kim E, Hwang J, Kim MJ. Liver fibrosis: stretched exponential model outperforms monoexponential and bi-exponential models of diffusion-weighted MRI. Eur Radiol 2018;28:2812-2822

33. Goo HW, Ra YS. Advanced MRI for pediatric brain tumors with emphasis on clinical benefits. Korean J Radiol 2017;18:194207

34. Saito K, Tajima Y, Harada TL. Diffusion-weighted imaging of the liver: current applications. World J Radiol 2016;8:857-867

35. Jerome NP, Miyazaki K, Collins DJ, Orton MR, d'Arcy JA, Wallace $T$, et al. Repeatability of derived parameters from histograms following non-Gaussian diffusion modelling of diffusion-weighted imaging in a paediatric oncological cohort. Eur Radiol 2017;27:345-353

36. Sui Y, Wang H, Liu G, Damen FW, Wanamaker C, Li Y, et al. Differentiation of low- and high-grade pediatric brain tumors with high b-value diffusion-weighted MR imaging and a fractional order calculus model. Radiology 2015;277:489-496

37. Binkovitz LA, El-Youssef M, Glaser KJ, Yin M, Binkovitz AK, Ehman RL. Pediatric MR elastography of hepatic fibrosis: principles, technique and early clinical experience. Pediatr Radiol 2012;42:402-409

38. Serai SD, Towbin AJ, Podberesky DJ. Pediatric liver MR elastography. Dig Dis Sci 2012;57:2713-2719

39. Venkatesh SK, Ehman RL. Magnetic resonance elastography of liver. Magn Reson Imaging Clin N Am 2014;22:433-446

40. Wagner M, Besa C, Bou Ayache J, Yasar TK, Bane 0, Fung M, et al. Magnetic resonance elastography of the liver: qualitative and quantitative comparison of gradient echo and spin echo echoplanar imaging sequences. Invest Radiol 2016;51:575581

41. Serai SD, Dillman JR, Trout AT. Spin-echo echo-planar imaging MR elastography versus gradient-echo MR elastography for assessment of liver stiffness in children and young adults suspected of having liver disease. Radiology 2017;282:761770

42. Manduca A, Oliphant TE, Dresner MA, Mahowald JL, Kruse $\mathrm{SA}$, Amromin E, et al. Magnetic resonance elastography: non-invasive mapping of tissue elasticity. Med Image Anal 2001;5:237-254

43. Yin M, Talwalkar JA, Glaser KJ, Venkatesh SK, Chen J, Manduca $A$, et al. Dynamic postprandial hepatic stiffness augmentation assessed with MR elastography in patients with chronic liver disease. AJR Am J Roentgenol 2011;197:64-70

44. Serai SD, Obuchowski NA, Venkatesh SK, Sirlin CB, Miller FH, Ashton E, et al. Repeatability of MR elastography of liver: a meta-analysis. Radiology 2017;285:92-100

45. Joshi M, Dillman JR, Towbin AJ, Serai SD, Trout AT. MR elastography: high rate of technical success in pediatric and young adult patients. Pediatr Radiol 2017;47:838-843

46. Trout AT, Serai S, Mahley AD, Wang H, Zhang Y, Zhang B, et al. Liver stiffness measurements with MR elastography: agreement and repeatability across imaging systems, field strengths, and pulse sequences. Radiology 2016;281:793-804

47. Gaddikeri S, Gaddikeri RS, Tailor T, Anzai Y. Dynamic contrastenhanced MR imaging in head and neck cancer: techniques and clinical applications. AJNR Am J Neuroradiol 2016;37:588595

48. Khalifa F, Soliman A, El-Baz A, Abou El-Ghar M, El-Diasty T, Gimel'farb G, et al. Models and methods for analyzing DCEMRI: a review. Med Phys 2014;41:124301

49. Azahaf M, Haberley M, Betrouni N, Ernst 0, Behal H, Duhamel $A$, et al. Impact of arterial input function selection on the accuracy of dynamic contrast-enhanced MRI quantitative analysis for the diagnosis of clinically significant prostate cancer. J Magn Reson Imaging 2016;43:737-749 
50. Jahng GH, Li KL, Ostergaard L, Calamante F. Perfusion magnetic resonance imaging: a comprehensive update on principles and techniques. Korean J Radiol 2014;15:554-577

51. Do RK, Rusinek H, Taouli B. Dynamic contrast-enhanced MR imaging of the liver: current status and future directions. Magn Reson Imaging Clin N Am 2009;17:339-349

52. Wu Z, Cheng ZL, Yi ZL, Xie MW, Zeng H, Lu LJ, et al. Assessment of nonalcoholic fatty liver disease in rats using quantitative dynamic contrast-enhanced MRI. J Magn Reson Imaging 2017; 45:1485-1493

53. Li Z, Sun J, Chen L, Huang N, Hu P, Hu X, et al. Assessment of liver fibrosis using pharmacokinetic parameters of dynamic contrast-enhanced magnetic resonance imaging. J Magn Reson Imaging 2016;44:98-104

54. Paldino MJ, Barboriak DP. Fundamentals of quantitative dynamic contrast-enhanced MR imaging. Magn Reson Imaging Clin N Am 2009;17:277-289

55. Hyodo T, Yada N, Hori M, Maenishi 0, Lamb P, Sasaki K, et al. Multimaterial decomposition algorithm for the quantification of liver fat content by using fast-kilovolt-peak switching dual-energy CT: clinical evaluation. Radiology 2017;283:108118

56. Siegel MJ, Curtis WA, Ramirez-Giraldo JC. Effects of dualenergy technique on radiation exposure and image quality in pediatric body CT. AJR Am J Roentgenol 2016;207:826-835

57. Leschied JR, Dillman JR, Bilhartz J, Heider A, Smith EA, Lopez MJ. Shear wave elastography helps differentiate biliary atresia from other neonatal/infantile liver diseases. Pediatr Radiol 2015;45:366-375

58. Wang X, Qian L, Jia L, Bellah R, Wang N, Xin Y, et al. Utility of shear wave elastography for differentiating biliary atresia from infantile hepatitis syndrome. $\mathrm{J}$ Ultrasound Med 2016;35:1475-1479

59. Zhou LY, Jiang $H$, Shan QY, Chen D, Lin XN, Liu BX, et al. Liver stiffness measurements with supersonic shear wave elastography in the diagnosis of biliary atresia: a comparative study with grey-scale US. Eur Radiol 2017;27:3474-3484

60. Lee MJ, Kim MJ, Yoon CS, Han SJ, Park YN. Evaluation of liver fibrosis with $\mathrm{T} 2$ relaxation time in infants with cholestasis: comparison with normal controls. Pediatr Radiol 2011;41:350354

61. Mo YH, Jaw FS, Ho MC, Wang YC, Peng SS. Hepatic ADC value correlates with cirrhotic severity of patients with biliary atresia. Eur J Radiol 2011;80:e253-e257

62. Peng SS, Jeng YM, Hsu WM, Yang JC, Ho MC. Hepatic ADC map as an adjunct to conventional abdominal MRI to evaluate hepatic fibrotic and clinical cirrhotic severity in biliary atresia patients. Eur Radiol 2015;25:2992-3002

63. Shin HJ, Yoon H, Kim MJ, Han SJ, Koh H, Kim S, et al. Liver intravoxel incoherent motion diffusion-weighted imaging for the assessment of hepatic steatosis and fibrosis in children. World J Gastroenterol 2018;24:3013-3020

64. Franchi-Abella S, Corno L, Gonzales E, Antoni G, Fabre $M$, Ducot $B$, et al. Feasibility and diagnostic accuracy of supersonic shear-wave elastography for the assessment of liver stiffness and liver fibrosis in children: a pilot study of 96 patients. Radiology 2016;278:554-562

65. Kim DW, Yoon HM, Jung AY, Lee JS, Oh SH, Kim KM, et al. Diagnostic performance of ultrasound elastography for evaluating portal hypertension in children: a systematic review and meta-analysis. J Ultrasound Med 2019;38:747-759

66. Hwang JY, Yoon HM, Kim JR, Lee JS, Jung AY, Kim KM, et al. Diagnostic performance of transient elastography for liver fibrosis in children: a systematic review and meta-analysis. AJR Am J Roentgenol 2018;211:W257-W266

67. Burak Özkan M, Bilgici MC, Eren E, Caltepe G. Diagnostic accuracy of point shear wave elastography in the detection of portal hypertension in pediatric patients. Diagn Interv Imaging 2018;99:151-156

68. Singh S, Venkatesh SK, Wang Z, Miller FH, Motosugi U, Low $\mathrm{RN}$, et al. Diagnostic performance of magnetic resonance elastography in staging liver fibrosis: a systematic review and meta-analysis of individual participant data. Clin Gastroenterol Hepatol 2015;13:440-451.e6

69. Xanthakos SA, Podberesky DJ, Serai SD, Miles L, King EC, Balistreri WF, et al. Use of magnetic resonance elastography to assess hepatic fibrosis in children with chronic liver disease. J Pediatr 2014;164:186-188

70. Yoon H, Shin HJ, Kim MJ, Han SJ, Koh H, Kim S, et al. Predicting gastroesophageal varices through spleen magnetic resonance elastography in pediatric liver fibrosis. World $\mathrm{J}$ Gastroenterol 2019;25:367-377

71. Erdem Toslak I, Lim-Dunham JE, Joyce C, Marbella ME. A practical approach to quantitative grayscale ultrasound analysis of hepatic steatosis in pediatric patients using a picture archiving and communication system-based tool. J Ultrasound Med 2018;37:2395-2403

72. Schwimmer JB, Middleton MS, Behling C, Newton KP, Awai $\mathrm{HI}$, Paiz MN, et al. Magnetic resonance imaging and liver histology as biomarkers of hepatic steatosis in children with nonalcoholic fatty liver disease. Hepatology 2015;61:18871895

73. Achmad E, Yokoo T, Hamilton G, Heba ER, Hooker JC, Changchien $C$, et al. Feasibility of and agreement between MR imaging and spectroscopic estimation of hepatic proton density fat fraction in children with known or suspected nonalcoholic fatty liver disease. Abdom Imaging 2015;40:3084-3090

74. Armstrong T, Ly KV, Murthy S, Ghahremani S, Kim GHJ, Calkins $\mathrm{KL}$, et al. Free-breathing quantification of hepatic fat in healthy children and children with nonalcoholic fatty liver disease using a multi-echo 3-D stack-of-radial MRI technique. Pediatr Radiol 2018;48:941-953

75. Koh H, Kim S, Kim MJ, Kim HG, Shin HJ, Lee MJ. Hepatic fat quantification magnetic resonance for monitoring treatment response in pediatric nonalcoholic steatohepatitis. World J Gastroenterol 2015;21:9741-9748

76. Powell EE, Ali A, Clouston AD, Dixon JL, Lincoln DJ, 
Purdie DM, et al. Steatosis is a cofactor in liver injury in hemochromatosis. Gastroenterology 2005;129:1937-1943

77. Yu H, Shimakawa A, McKenzie CA, Brodsky E, Brittain JH, Reeder SB. Multiecho water-fat separation and simultaneous R2* estimation with multifrequency fat spectrum modeling. Magn Reson Med 2008;60:1122-1134

78. Wang X, Hernando D, Reeder SB. Sensitivity of chemical shiftencoded fat quantification to calibration of fat MR spectrum. Magn Reson Med 2016;75:845-851

79. Manning P, Murphy P, Wang K, Hooker J, Wolfson T, Middleton MS, et al. Liver histology and diffusion-weighted MRI in children with nonalcoholic fatty liver disease: a MAGNET study. J Magn Reson Imaging 2017;46:1149-1158

80. Trout AT, Sheridan RM, Serai SD, Xanthakos SA, Su W, Zhang $B$, et al. Diagnostic performance of MR elastography for liver fibrosis in children and young adults with a spectrum of liver diseases. Radiology 2018;287:824-832

81. Dillman JR, Trout AT, Costello EN, Serai SD, Bramlage KS, Kohli R, et al. Quantitative liver MRI-biopsy correlation in pediatric and young adult patients with nonalcoholic fatty liver disease: can one be used to predict the other? AJR Am J Roentgenol 2018;210:166-174

82. Park SH, Lee SS, Sung JY, Na K, Kim HJ, Kim SY, et al. Noninvasive assessment of hepatic sinusoidal obstructive syndrome using acoustic radiation force impulse elastography imaging: a proof-of-concept study in rat models. Eur Radiol 2018;28:2096-2106

83. Dietrich CF, Averkiou MA, Correas JM, Lassau N, Leen E, Piscaglia F. An EFSUMB introduction into dynamic contrastenhanced ultrasound (DCE-US) for quantification of tumour perfusion. Ultraschall Med 2012;33:344-351

84. Tian WS, Lin MX, Zhou LY, Pan FS, Huang GL, Wang W, et al. Maximum value measured by 2-D shear wave elastography helps in differentiating malignancy from benign focal liver lesions. Ultrasound Med Biol 2016;42:2156-2166

85. Park HS, Kim YJ, Yu MH, Jung SI, Jeon HJ. Shear wave elastography of focal liver lesion: intraobserver reproducibility and elasticity characterization. Ultrasound Q 2015;31:262-271

86. Chiorean L, Cui XW, Tannapfel A, Franke D, Stenzel M, Kosiak $W$, et al. Benign liver tumors in pediatric patients - Review with emphasis on imaging features. World J Gastroenterol 2015;21:8541-8561

87. Masand PM. Magnetic resonance imaging features of common focal liver lesions in children. Pediatr Radiol 2018;48:12341244

88. Zhang W, Chen HJ, Wang ZJ, Huang W, Zhang LJ. Dynamic contrast enhanced MR imaging for evaluation of angiogenesis of hepatocellular nodules in liver cirrhosis in N-nitrosodiethylamine induced rat model. Eur Radiol 2017;27:2086-2094 